\section{Vitalkapazitätsverlust bei systemischer Sklerose mit ILD verlangsamen}

\author{
Distler, O et al. Nintedanib for Systemic Sclerosis \\ Associated Interstitial Lung Disease. N Engl J Med \\ 2019; 380: 2518-2528. doi: 10.1056/ \\ NEJMoa1903076.
}

Eine häufige Manifestation und eine führende erkrankungsbedingte Todesursache bei systemischer Sklerose ist eine interstitielle Lungenerkrankung (ILD). Präklinische Untersuchungen haben auf einen antifibrotischen und antiinflammatorischen Effekt des für die Therapie der idiopathischen Lungenfibrose (IPF) zugelassenen Tyrosinkinase-Inhibitors (TKI) Nintedanib in dieser Situation hingewiesen.

Die mit systemischer Sklerose assoziierte ILD weist zwar andere Trigger auf als die IPF, die pathophysiologischen Prozesse umfassen aber bei beiden Erkrankungen die Transformation von Fibroblasten in einen myofibroblastischen Phänotyp und eine exzessive Ablagerung von extrazellulärer Matrix. Daher untersuchte eine große randomisierte, Placebo-kontrollierte Studie, ob Nintedanib auch bei der mit systemischer Sklerose assoziierten ILD wirksam und sicher ist. Wie Oliver Distler von der Abtei-

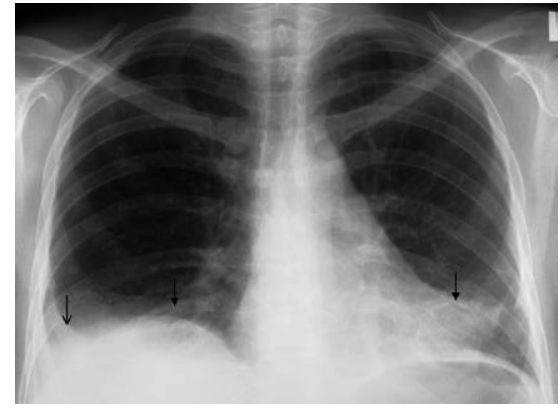

Systemischer Lupus erythematodes mit Lungenbeteiligung und Pleuraerguss rechts ( $\downarrow$ ). Quelle: Krug K, Kamm K, Waßermann $\mathrm{K}$ et al. Kollagenosen. In: Krug K, Hrsg. Thoraxdiagnostik. 1. Auflage. Stuttgart: Thieme; 2004

lung für Rheumatologie an der Universität Basel und Koautoren des Senscis-Studienteams berichten, wurden Patienten mit systemischer Sklerose eingeschlossen, die in den letzten 7 Jahren das erste Non-Raynaud-Symptom der Erkrankung entwickelt hatten und in der hochauflösenden Computertomografie eine Fibrose in mindestens $10 \%$ der Lunge aufwiesen. Patienten mit pulmonaler Hypertonie wurden ausgeschlossen.

Randomisiert erhielten die Studienteilnehmer entweder Nintedanib in einer Dosis von $150 \mathrm{mg}$ oral zweimal täglich oder Placebo. Primärer Endpunkt der 52 Wochen dauernden Studie war die auf ein Jahr bezogene Abnahme der forcierten Vitalkapazität (FVC). Wichtige sekundäre Endpunkte betrafen den modifizierten Rodnan-Hautscore zur Erfassung der Hautsymptome der systemischen Sklerose und die krankheitsbezogene Lebensqualität, ermittelt mit dem St. George's Respiratory Questionnaire (SGRQ) in Woche 52.

\section{Ergebnisse}

Insgesamt 576 Patienten hatten in den beiden Gruppen mindestens eine Dosis der Prüfmedikation oder Placebo erhalten. Von diesen Patienten wiesen bei Studieneinschluss gut die Hälfte (51,9\%) eine diffuse kutane systemische Sklerose auf, knapp die Hälfte (48,4 \%) wurden mit Mycophenolat behandelt. 
Die Analyse des primären Endpunkts zeigte einen klaren Vorteil der Therapie mit Nintedanib gegenüber Placebo: Die Abnahme der FVC lag mit dem TKI bei $-52,4$ ml pro Jahr, in der Placebogruppe bei -93,3 ml pro Jahr. Die Autoren betonen, dass die Placebogruppe in dieser Studie gegenüber anderen Therapiestudien besonders gut abgeschnitten hat. Dennoch führte die Nintedanib-Therapie zu einer um 41,0 ml pro Jahr geringeren FVC-Abnahme (95\% Konfidenzintervall $[\mathrm{KI}] 2,9-79,0 ; \mathrm{p}=0,04)$. Sensitivitätsanalysen zeigten allerdings eine Unsicherheit bezüglich des p-Werts aufgrund von fehlenden Daten (Spanne von 0,06 bis $0,10)$. Zudem beeinflusste Nintedanib die Hautsymptome nicht, und auch die Lebensqualität in Woche 52 war in der Verumgruppe nicht signifikant besser als in der Placebogruppe. Die häufigste Nebenwirkung von Nintedanib waren Durchfälle. Sie betrafen drei Viertel der Patienten (75,7 \%), im Placebo-Arm traten bei 31,6 \% der Patienten Diarrhoen auf.

FAZIT

Nintedanib hat das Potenzial, die Abnahme der FVC bei mit systemischer Sklerose assoziierter ILD ähnlich gut wie bei IPF zu verringern. Ein krankheitsmodifizierender Effekt auf die systemische Sklerose hat der TKI aber nicht, betonen die Autoren. Auch fand sich kein positiver Effekt auf patientenberichtete Endpunkte. Die Komedikation mit Mycophenolat beeinflusste die Abnahme der FVC in der Verum- und in der Placebogruppe und demonstriert damit einen eigenen Effekt auf die FVC-Abnahme.

Friederike Klein, München 